\title{
Nucleosynthesis in AGB Stars
}

\author{
John Lattanzio \\ Dept of Mathematics and Statistics, Monash University, Australia \\ Institute of Astronomy, Madingley Rd, Cambridge, England \\ Manuel Forestini \\ Laboratoire d'Astrophysique, Université Joseph Fourier, Grenoble, \\ France
}

\begin{abstract}
We give a qualitative review of the nucleosynthesis occurring in AGB stars. We summarise some new calculations of intermediate mass stars which include all thermal pulses until the star is about to leave the AGB, as well as a detailed nucleosynthesis network. We will show that hot bottom burning delays, rather than prevents, the formation of carbon stars; those that form are not optically visible.
\end{abstract}

\section{Introduction}

Although only a brief phase compared to the overall stellar lifetime, the Asymptotic Giant Branch is crucial because it is here that stars experience substantial nucleosynthesis, which is the topic of this review.

The AGB phase is the last phase of evolution for low and intermediate mass stars (about $1-8 M_{\odot}$ ). The evolution leading up to this phase has been discussed in detail in Lattanzio \& Boothroyd (1997) and Lattanzio (1998). Briefly, stars begin by burning hydrogen in their cores. Following the exhaustion of their central hydrogen supply, they become red-giants. During this phase the stars develop a deep convective envelope which extends inwards and can reach into regions where partial hydrogen burning has taken place. This is called the "first dredge-up" event, and it results in polluting the stellar surface with the products of hydrogen burning.

The ascent of the giant branch is terminated by ignition of the central helium supply, either degenerately in a core flash (in the case of low mass stars, below about $2.5 M_{\odot}$ ) or non-degenerately (for higher masses). The subsequent evolution is characterized by helium burning in a convective core and a steadily advancing hydrogen burning shell. For low mass stars the evolution is complicated by semiconvection, but this is a detail we do not need for our present purposes. The fusion of helium produces ${ }^{12} \mathrm{C}$, and this carbon is in turn subject to alpha capture to form ${ }^{16} \mathrm{O}$. Eventually the helium supply is totally consumed, leaving a core of carbon and oxygen (the exact proportions of which depend on the infamously uncertain rate for the ${ }^{12} \mathrm{C}(\alpha, \gamma){ }^{16} \mathrm{O}$ reaction). In any event, 


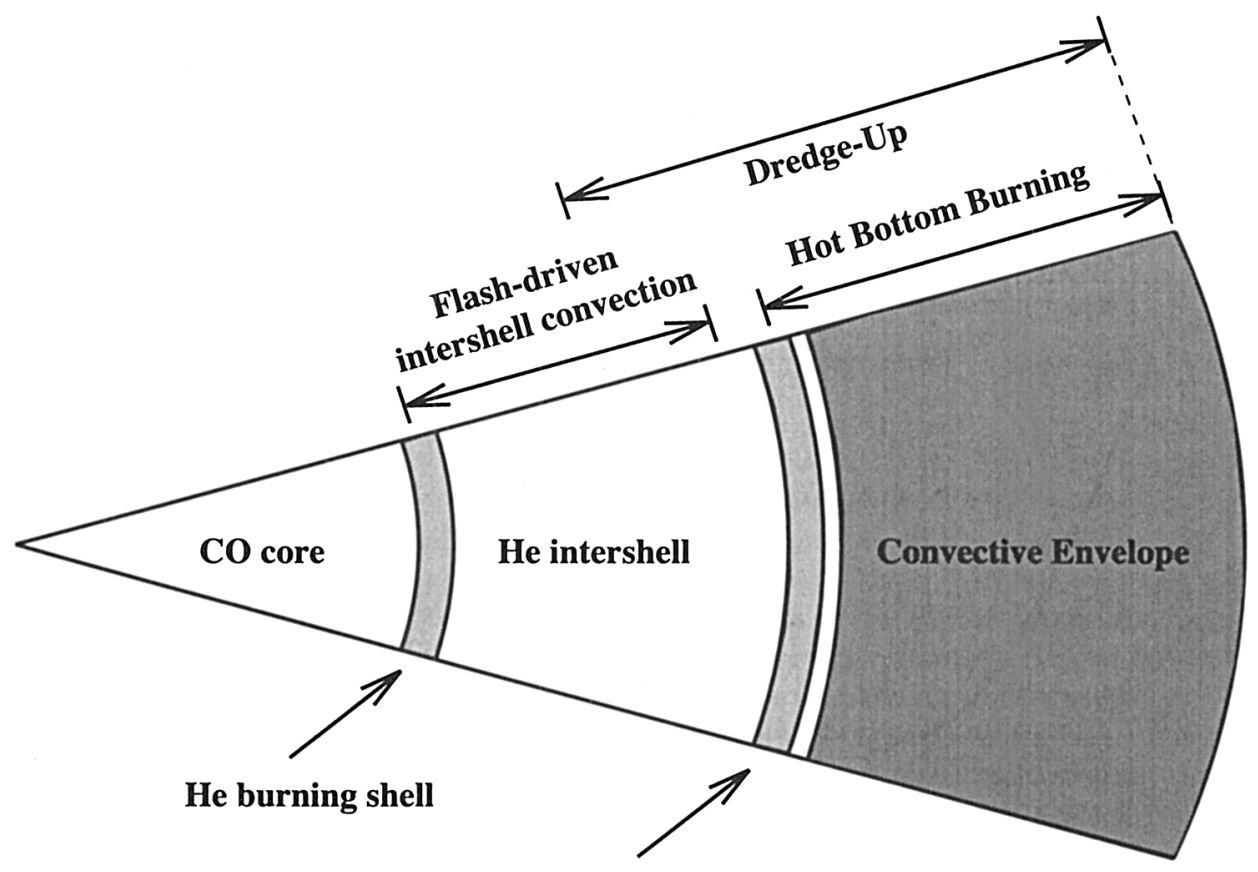

H burning shell

Figure 1. Schematic of the interior of an AGB star.

following the exhaustion of central helium the star begins to ascend the giant branch again. It is now called the "second" or "asymptotic" giant branch.

When a star reaches the AGB it has the structure shown in Fig. 1. If its mass is above about $4 M_{\odot}$ the shifting of helium burning from the core to a shell causes the extinguishing of the hydrogen shell. In lower mass stars this stops the inward advance of the convective envelope, but in more massive stars the envelope penetrates the extinct hydrogen shell, and products of hydrogen burning are mixed to the stellar surface, in the "second dredge-up" episode. Note that low mass stars do not experience the second dredge-up.

Perhaps the most important aspect of AGB stars lies in the nucleosynthesis which occurs during their evolution. During this phase they show an electron degenerate carbon-oxygen core, and two nuclear shells: one burning helium and one burning hydrogen. The subsequent nucleosynthesis comes from two sources: the thermal instability of the helium shell (known as "thermal pulses" or "shell flashes") and, for masses above about $5 M_{\odot}$, nuclear burning at the bottom of the convective envelope (known as "hot bottom burning", hereafter $\mathrm{HBB})$.

\section{Thermally pulsing AGB evolution}

For most of the evolution on the AGB, the helium shell is largely inactive. But periodically the shell suffers a thermal runaway and generates enormous 
quantities of energy, at rates up to $10^{8} L_{\odot}$ or so, for short periods of time (of order a year). This energy release results in a "flash-driven convection zone" which extends from the helium shell almost to the hydrogen shell, as shown in Fig. 1. This mixes the ${ }^{12} \mathrm{C}$ produced by the helium shell throughout this convective region. As the helium flash dies down, the energy deposited in the star causes a substantial expansion and cooling. The hydrogen shell is extinguished and the convective envelope reaches in beyond its old location, and (for later pulses) into the carbon-rich region left behind by the flash convection. This mixes carbon to the surface of the star, and is known as the "third dredge-up". It is responsible for the production of $\mathrm{N}$-type carbon stars. As the star now contracts again, the hydrogen shell is re-ignited and the star begins an extended "interpulse" phase of order $10^{4}$ years (but depending primarily on the mass of the hydrogen exhausted core, hereafter called the core-mass). During this period the hydrogen shell provides essentially all of the star's luminosity. Eventually there is another flash, and the cycle repeats. We will give a qualitative summary of AGB nucleosynthesis below, but details may be found in Forestini \& Charbonnel (1997).

Another important consequence of thermal pulses is the production of elements beyond iron, produced by slow neutron capture, and known as $s$-process elements. For a detailed review see Gallino, Busso \& Lugaro (1997). For our purposes here, we simply note that some partial mixing of hydrogen from the envelope into the carbon-rich intershell region is required. These protons are captured by ${ }^{12} \mathrm{C}$ to produce ${ }^{13} \mathrm{C}$ which later releases neutrons via the ${ }^{13} \mathrm{C}(\alpha, \mathrm{n}){ }^{16} \mathrm{O}$ reaction. Early calculations by Iben \& Renzini $(1982 a, b)$ found that a small semiconvective zone formed at the bottom of the convective envelope, during the dredge-up phase. But other calculations have failed to reproduce the process. (Normal, complete mixing will not suffice, incidentally, because the number of protons must be small, or else the $\mathrm{CN}$ cycle will go to completion and produce ${ }^{14} \mathrm{~N}$ rather than ${ }^{13} \mathrm{C}$.) Nevertheless it, or something very much like it, almost certainly occurs. Observations of these stars clearly show the $s$-process enhancements that these ideas would predict. Quantitative calculations are performed by Gallino and co-workers (e.g. see Gallino, Busso \& Lugaro 1997) and are very successful, although the amount of ${ }^{13} \mathrm{C}$ present is a parameter which must be specified in advance. Other possible mixing mechanisms are overshoot, and partial mixing (see Herwig et al. 1997), or possibly something like shear mixing.

A related issue is the production of ${ }^{19} \mathrm{~F}$ within these stars. Jorissen, Smith \& Lambert (1992) discovered that the ${ }^{19} \mathrm{~F} /{ }^{16} \mathrm{O}$ ratio in $\mathrm{AGB}$ stars increases with the ${ }^{12} \mathrm{C} /{ }^{16} \mathrm{O}$ ratio, which implicates thermal pulses in the origin of this ${ }^{19} \mathrm{~F}$. They investigated many possible scenarios, and this was followed by Forestini et al. (1992) who investigated the most promising scenario in more detail, and to whom we defer for details (see also Mowlavi, Jorissen \& Arnould 1996). The mechanism is shown in Fig. 2. Here, some ${ }^{13} \mathrm{C}$ produces neutrons via the ${ }^{13} \mathrm{C}(\alpha, \mathrm{n}){ }^{16} \mathrm{O}$ reaction, and some of these neutrons are captured by ${ }^{14} \mathrm{~N}$ to produce ${ }^{14} \mathrm{C}$ and protons. These protons, plus possibly some from ${ }^{26} \mathrm{Al}(\mathrm{n}, \mathrm{p})^{26} \mathrm{Mg}$, are then captured by ${ }^{18} \mathrm{O}$ and the sequence ${ }^{18} \mathrm{O}(\mathrm{p}, \alpha){ }^{15} \mathrm{~N}(\alpha, \gamma){ }^{19} \mathrm{~F}$ produces the observed ${ }^{19} \mathrm{~F}$, which is then dredged to the surface in the usual way following the pulse. For all except those stars with the highest abundances of ${ }^{19} \mathrm{~F}$ it appears that the amount of ${ }^{13} \mathrm{C}$ left from the $\mathrm{CN}$ cycling $\mathrm{H}$-shell is sufficient. 


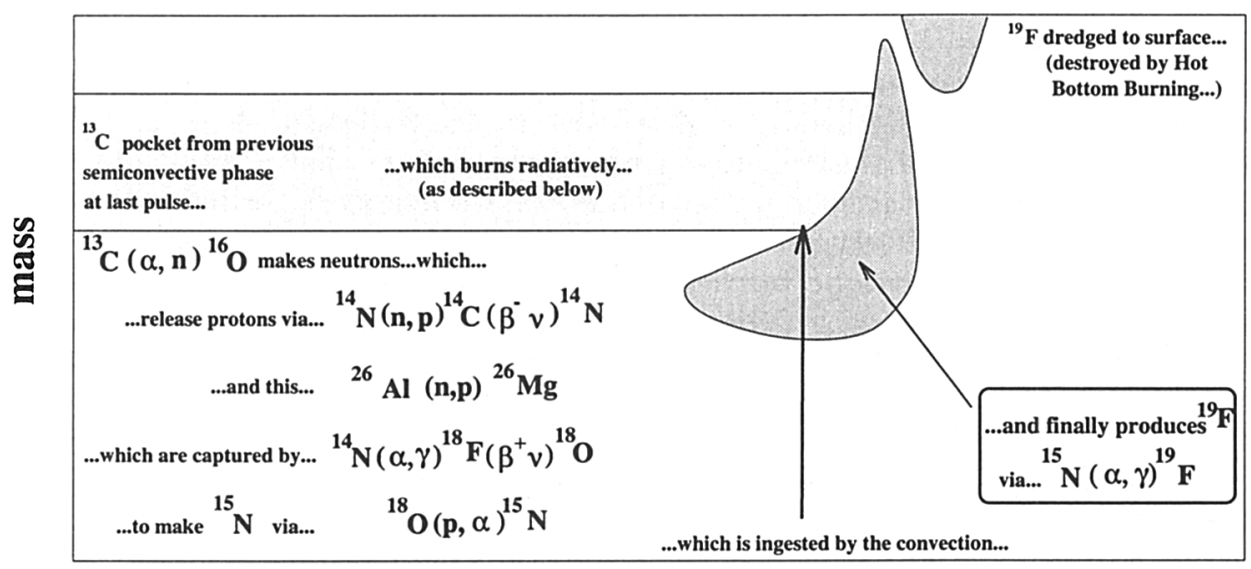

time

Figure 2. Production of ${ }^{19} \mathrm{~F}$ in AGB stars.

Yet for those stars showing more enhanced ${ }^{19} \mathrm{~F}$ we may need to invoke some extra-mixing, just as is required for the $s$-process production.

For stars above about $5 M_{\odot}$ a second important phenomenon is the occurrence of hot bottom burning. Here the convective envelope is so deep that it penetrates into the top of the hydrogen burning shell, so that nucleosynthesis actually occurs in the envelope of the star. Temperatures can reach as high as almost $10^{8} \mathrm{~K}$. This results in the activation of the $\mathrm{CN}$ cycle within the envelope, and the consequent processing of ${ }^{12} \mathrm{C}$ into ${ }^{13} \mathrm{C}$ and ${ }^{14} \mathrm{~N}$. This prevents the star from becoming a carbon star, because the primary carbon dredged to the envelope is then transmuted into nitrogen, keeping the $\mathrm{C} / \mathrm{O}$ ratio less than unity (e.g. Boothroyd et al. 1993).

Perhaps the most striking consequence of hot bottom burning is the production of ${ }^{7} \mathrm{Li}$ by the Cameron-Fowler Mechanism. Sackmann \& Boothroyd (1992) showed that ${ }^{7} \mathrm{Li}$ was produced as soon as the temperature $T_{b c e}$ at the bottom of the convective envelope exceeded $50 \times 10^{6} \mathrm{~K}$, and produced abundances which match the Magellanic Cloud observations very nicely (see below). Another consequence of $\mathrm{HBB}$ is the substantial production of ${ }^{26} \mathrm{Al}$ by the $\mathrm{Mg}-\mathrm{Al}$ cycle. The amount produced can be substantial, and this is implicated in explaining the ${ }^{26} \mathrm{Al} /{ }^{27} \mathrm{Al}$ isotopic ratios seen in meteorite grains (for a review please see Zinner 1997).

\section{Complete AGB evolution for intermediate mass stars}

We have recently completed detailed evolutionary calculations for stars of intermediate masses from the ZAMS through to the end of the AGB evolution (Frost 1998). These calculations covered three compositions: $\mathrm{Z}=0.004$ (corresponding to the Small Magellanic Cloud), $\mathrm{Z}=0.008$ (Large Magellanic Cloud) and $\mathrm{Z}=0.02$ (solar neighbourhood). For each composition we have considered three masses, $M / M_{\odot}=4,5$ and 6 . Subsequent nucleosynthesis has been calculated 


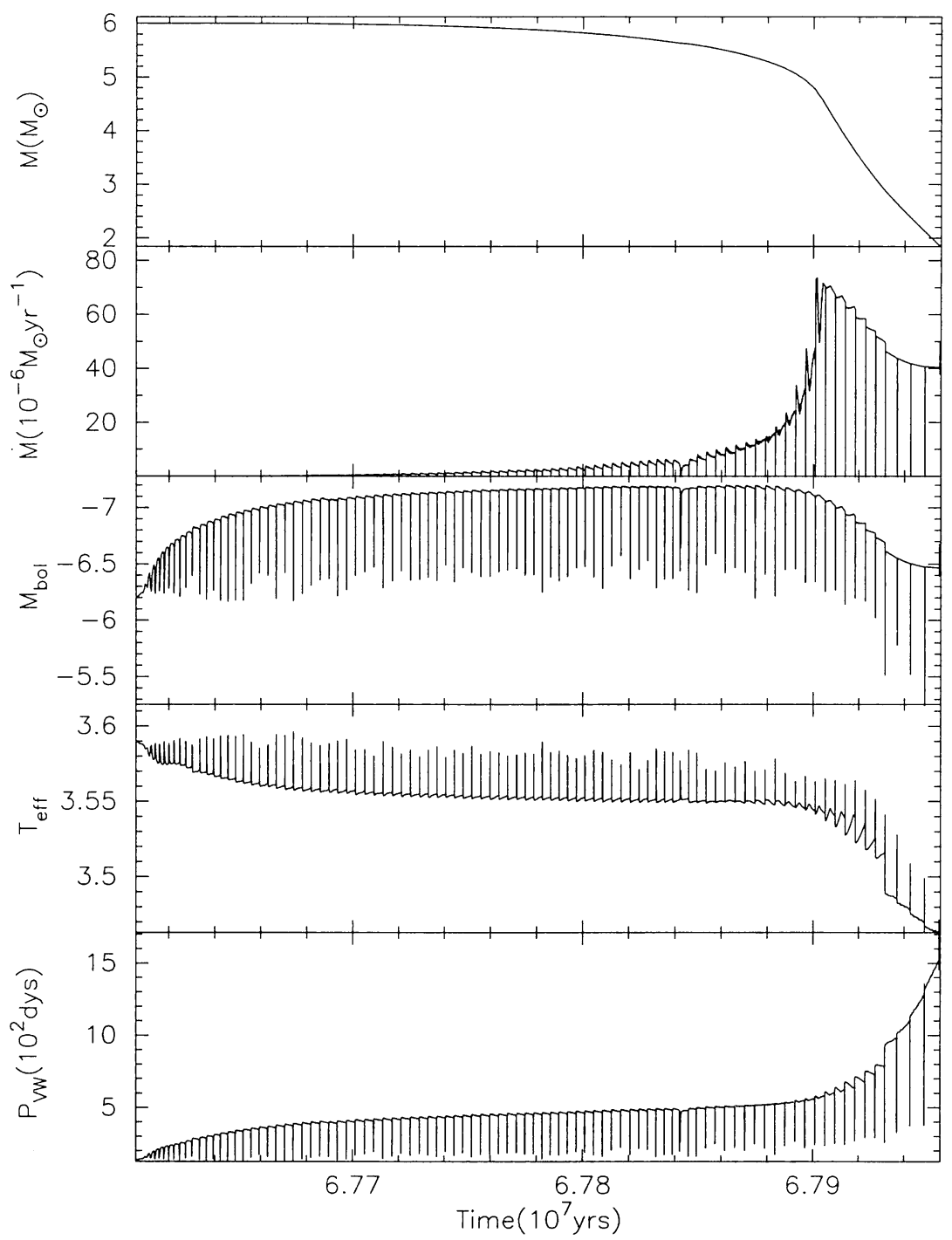

Figure 3. AGB evolution for a $6 M_{\odot}$ model with $Z=0.004$. The upper panel shows the variation in total mass; the second panel shows the variation in the mass-loss rate; next is the bolometric magnitude, then the effective temperature; finally we show the period as calculated by the formula of Vassiliadis \& Wood (1993). 

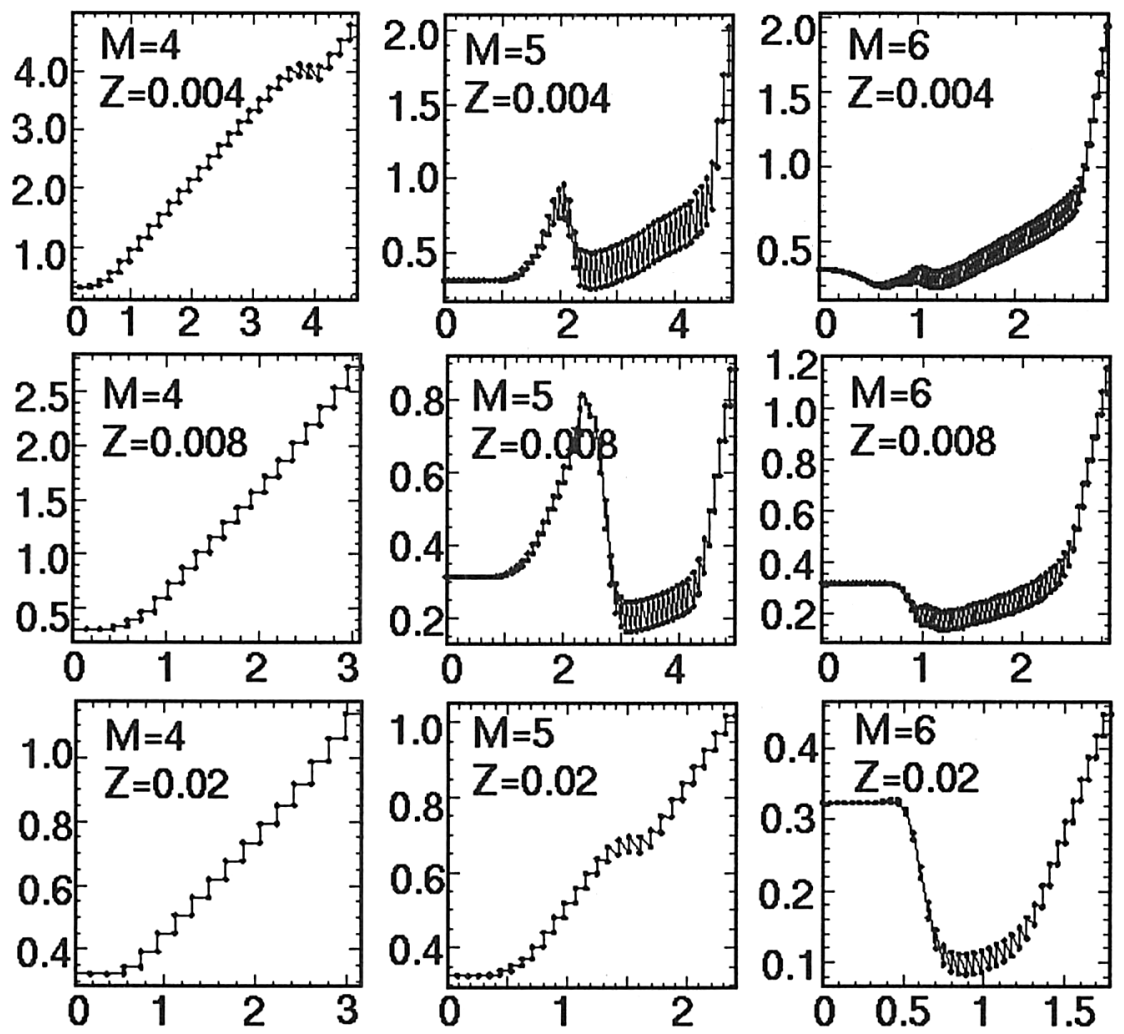

Figure 4. Surface value of $\mathrm{C} / \mathrm{O}$ for the nine cases discussed in the text. The $x$-axis is the time elapsed (in $10^{5}$ years) since the first thermal pulse.

using the Monash University Nucleosynthesis Code (based on that of Cannon 1993). Results from these calculations have not yet been published, with the exception of one preliminary report (Lattanzio et al. 1996) and two short papers (Frost et al. 1998; Frost, Lattanzio \& Wood 1998). A brief summary will be given here, and details will be published elsewhere.

\subsection{Model calculations}

Models were calculated with the Mt Stromlo Stellar Structure Code, updated to include the OPAL opacities. Mass-loss was included using the prescription of Vassiliadis \& Wood (1993), but without the modification for $M$ above $2.5 M_{\odot}$. As an example of the AGB evolution, Fig. 3 shows a summary of the case for the $6 M_{\odot}$ model with $Z=0.004$. We note a crucial aspect of this evolution: mass-loss is relatively small until near the end of the evolution, when the rate becomes very high. We will see the consequences of this below. 

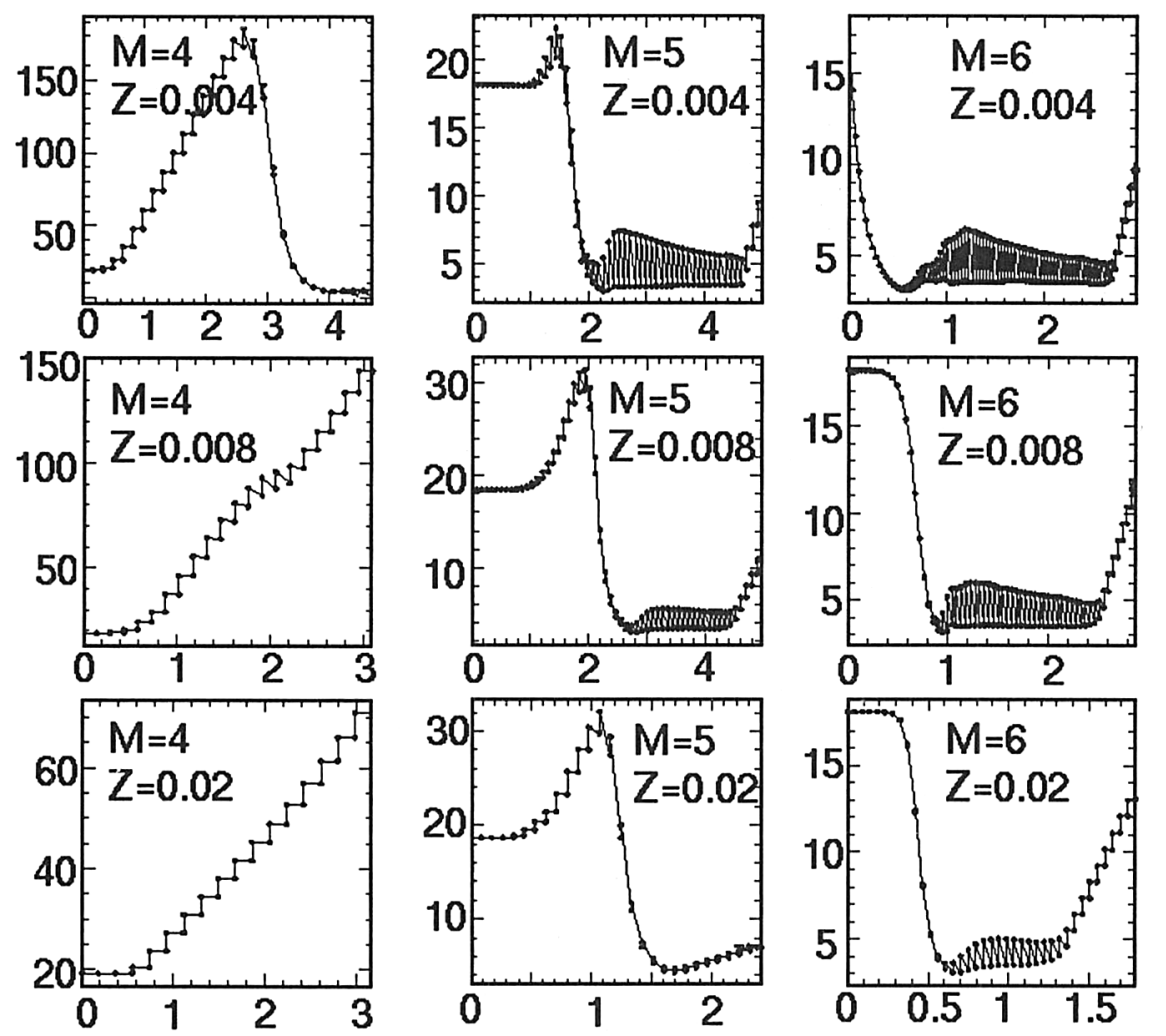

Figure 5. Same as Fig. 4 but for ${ }^{12} \mathrm{C} /{ }^{13} \mathrm{C}$.

The nucleosynthesis calculations include all elements up to sulphur and also a small $s$-process network based on the iron-peak. We have also developed the extrapolation code of Forestini \& Charbonnel (1997) to include the results of these detailed calculations. For many variables we have found it best to interpolate within envelope mass, rather than the traditional core-mass. Some of the results presented below actually come from the synthetic code (and agree with the detailed calculations to within $10 \%$ ).

\subsection{The brightest carbon stars}

Fig. 4 shows the surface $\mathrm{C} / \mathrm{O}$ ratio for the nine models considered. The repeated effects of dredge-up are clearly visible in the discontinuous changes in the ratio at each dredge-up event. The effect of $\mathrm{HBB}$ can also be seen as a smooth decrease in the ratio between dredge-up events. Note that there is no HBB in the three $4 M_{\odot}$ models, and the $\mathrm{C} / \mathrm{O}$ ratio increases monotonically for these cases (in fact, there is a small amount of HBB in the $Z=0.004$ case at a time of about $4 \times 10^{5}$ years).

The effects of $\mathrm{HBB}$ are clearly seen in the 5 and $6 M_{\odot}$ models. Consider firstly the $5 M_{\odot}$ cases, where the $\mathrm{HBB}$ is important at low metallicities (due to 

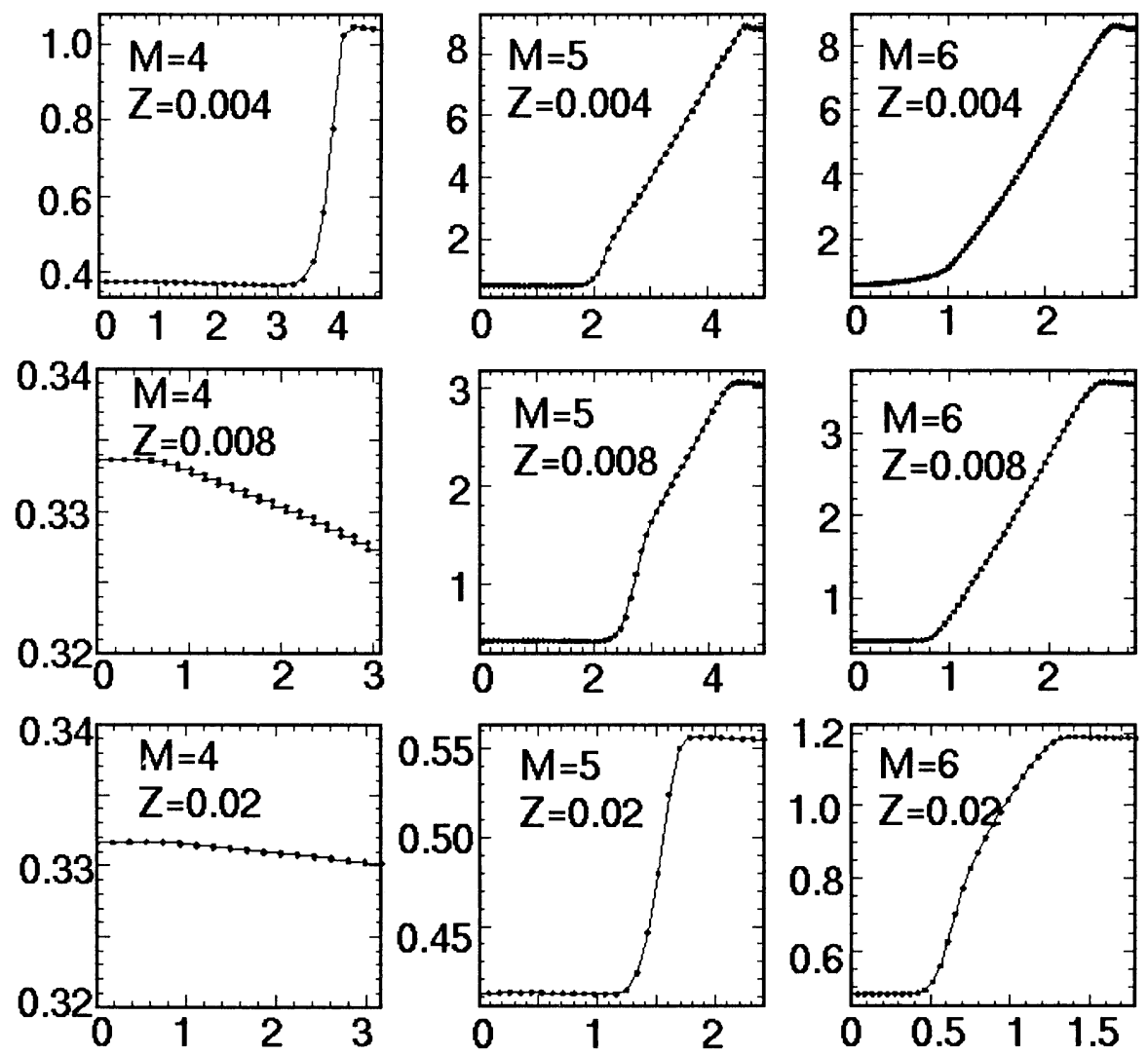

Figure 6. Same as Fig. 4 but for N/O.

higher temperatures in these cases). Although dredge-up begins in the M5Z004 ${ }^{1}$ case and produces a $\mathrm{C} / \mathrm{O}$ ratio almost unity, the start of $\mathrm{HBB}$ then reduces this value dramatically and keeps it below unity until an AGB age of about $4 \times 10^{5}$ years. This is also seen in the $\mathrm{M} 5 \mathrm{Z} 008$ case, where the $\mathrm{C} / \mathrm{O}$ ratio reaches 0.8 before $\mathrm{HBB}$ reduces it to about 0.2 . Even in the M5Z02 case we see the start of $\mathrm{HBB}$ at an AGB age of $1.7 \times 10^{5}$ years. Yet in each of these three cases the $\mathrm{C} / \mathrm{O}$ ratio finally increases. This is due to mass-loss, which decreases the envelope mass and hence the dilution of any material added by dredge-up.

The continued decreasing of the envelope mass finally reduces the weight of the envelope to such an extent that HBB is terminated. Yet dredge-up continues. This is a new result from these calculations, and it is very important for understanding the final stages of $\mathrm{AGB}$ evolution. Hence the $\mathrm{C} / \mathrm{O}$ ratio again begins to increase as ${ }^{12} \mathrm{C}$ is added to the envelope without being burned by HBB. This effect shows itself most dramatically when the mass decreases the

${ }^{1} \mathrm{MaZb}$ refers to the case with $M=a M_{\odot}$ and $Z=b$. 
most, which is near the end of the AGB lifetime (see Fig. 3). Note that HBB is quite extreme in the $6 M_{\odot}$ cases, where the $\mathrm{C} / \mathrm{O}$ ratio is kept very small (due to HBB) until the end of the star's lifetime, when it can indeed exceed unity. Thus we predict a population of bright $\left(M_{b o l} \simeq-6.6\right)$ carbon stars, but these would not be optically visible because of their high mass-loss. This result was reported in Frost et al. (1998) and seems to explain the infra-red population of carbon stars reported by van Loon et al. (1998) and earlier by Kastner et al. (1993).

HBB produces ${ }^{13} \mathrm{C}$ by the $\mathrm{CN}$ cycling at the bottom of the envelope. This is shown in Fig. 5. The timescales are such that the entire envelope is processed by $\mathrm{HBB}$ and this can result in the ${ }^{12} \mathrm{C} /{ }^{13} \mathrm{C}$ ratio reaching its equilibrium value of $3-3.5$, as is seen in many cases in Fig. 5 . In fact, one may use this ratio, with care, as an indicator of $\mathrm{HBB}$. An observed low value is strong evidence for $\mathrm{HBB}$, whereas a high value is evidence for dredge-up of ${ }^{12} \mathrm{C}$ without $\mathrm{HBB}$. Intermediate values are harder to interpret.

A very important consequence of $\mathrm{HBB}$ in these stars is the production of primary ${ }^{14} \mathrm{~N}$. This is shown in Fig. 6. Substantial enhancement factors can be obtained, especially for the more massive low metallicity models. These stars are likely to be an important source of ${ }^{14} \mathrm{~N}$ in the Galaxy, especially at earlier times when the metal content of intermediate mass stars is low.

HBB produces ${ }^{7} \mathrm{Li}$, which is therefore a good indicator of the presence of $\mathrm{HBB}$, although in the more massive and lower metallicity models we can find substantial Li production even before the first thermal pulse. Hence models such as M6Z004 begin their thermally-pulsing AGB with high $\mathrm{Li}$ abundances. Note also that when the initial supply of ${ }^{3} \mathrm{He}$ is used up, there is again a decrease of the $\mathrm{Li}$ content. The models agree very well with the observations of Smith \& Lambert $(1989,1990)$ and Smith et al. (1995) which show Magellanic Cloud AGB stars of $M_{b o l}$ between -6 and -7 to have ${ }^{2} \log \varepsilon\left({ }^{7} \mathrm{Li}\right)$ in the range 2.2 to 3.8 . This was also shown (first!) by Sackmann \& Boothroyd (1992).

During the evolution of the models discussed above, we found a different kind of thermal pulse would occasionally occur. This resulted from the prolonged occurrence of deep dredge-up on the AGB. This cools the intershell region and extinguishes the helium burning before the tail of the helium shell has burned fully, and results in a long tail extending deeply into the star, and experiencing partially degenerate conditions. Eventually this tail ignites in a "degenerate pulse" and produces a shell flash of unusually high temperatures, as reported in Frost, Lattanzio \& Wood (1998). The nucleosynthesis occurring in these cases is currently under investigation.

\section{Conclusion}

We have made an extensive investigation of the evolution and nucleosynthesis of intermediate mass stars. This is not to say that there are no uncertainties: the details of how to calculate dredge-up remain a serious problem. But we have the tools for a consistent investigation of the behaviour of the stars, given this caveat. Intermediate mass stars were investigated first, but it is well known

$\left.{ }^{2} \log \varepsilon\left({ }^{7} \mathrm{Li}\right) \equiv \log \left\{n\left({ }^{7} \mathrm{Li}\right) / n(\mathrm{H})\right\}+12\right)$. 
that the majority of the carbon stars and $s$-process enriched stars are of lower masses, about $1-3 M_{\odot}$. These are the subject of current work.

Acknowledgments. We are particularly grateful to the organisers for the meeting, and JL also wishes to thank them for their financial assistance.

\section{References}

Boothroyd A.I., Sackmann I.-J., Ahern S.C., 1993, ApJ 416, 762

Cannon R.C., 1993, MNRAS 263, 817

Forestini M., Charbonnel C., 1997, A\&AS 123, 241

Forestini M., et al., 1992, A\&A 261, 157

Frost C.A., 1998, Ph. D thesis, Monash University

Frost C.A., Lattanzio J.C., Wood P.R., 1998, ApJ 500, 355

Frost C.A., et al., 1998, A\&A 332, L17

Gallino R., Busso M., Lugaro M., 1997, in Astrophysical Implications of the Laboratory Study of Presolar Materials, T.J. Bernatowicz \& E. Zinner (eds.), AIP, p. 115

Herwig F., Blöcker T., Schönberner D., El Eid M., 1997, A\&A 324, L81

Iben I., Jr., Renzini A., 1982a, ApJ 259, L79

Iben I., Jr., Renzini A., 1982b, ApJ 263, L23

Jorissen A., Smith V.V., Lambert D.L., 1992, A\&A 261, 164

Kastner J.H., et al., 1993, A\&A 275, 163

Lattanzio J.C., 1998, in Stellar Evolution, Stellar Explosions and Galactic Chemical Evolution, A. Mezzacappa (ed.), IOP, p. 299

Lattanzio J.C., Boothroyd A.I., 1997, in Astrophysical Implications of the Laboratory Study of Presolar Materials, T. J. Bernatowicz \& E. Zinner (eds.), AIP, p. 85

Lattanzio J.C., Frost C.A., Cannon R.C., Wood P.R., 1996, Mem. Astro. Soc. Italia 67,729

Mowlavi N., Jorissen A., Arnould M., 1996, A\&A 311, 803

Sackmann I.-J., Boothroyd A.I., 1992, ApJ 392, L71

Smith V.V., Lambert D.L., 1989, ApJ 345, 375

Smith V.V., Lambert D.L., 1990, ApJ 361, L69

Smith V.V., Lambert D.L., Plez B., Lubowich D.A., 1995, ApJ 441, 735

van Loon J.Th., et al., 1998, A\&A 329, 169

Vassiliadis E., Wood P.R., 1993, ApJ 413, 641

Zinner E., 1997, in Astrophysical Implications of the Laboratory Study of Presolar Materials, T.J. Bernatowicz \& E. Zinner (eds.), AIP, p. 3 